\title{
Kahoot como herramienta de transición en las aulas invertidas. La aplicación en la docencia en Filosofía del Derecho
}

\author{
Crego, Jorge \\ Facultade de Dereito. Universidade da Coruña. ORCID: 0000-0001-7072-6569 \\ Universidad Internacional de La Rioja
}

\section{RESUMEN}

La materia de Filosofía del Derecho impartida en la Universidade da Coruña (UDC) incluye como tema 7 un estudio de las teorías de la justicia, basada en los materiales de Michael J. Sandel (Harvard). Dicho tema se desarrolla a través de las aulas de seminario. Siguiendo la propuesta del profesor estadounidense, dicho tema utiliza la metodología del aula invertida 0 flipped classroom. Este trabajo tiene como objetivo evaluar la introducción de recursos tecnológicos (Kahoot) para impulsar la correcta realización del trabajo autónomo fuera del aula asignado a la materia. A través de la realización de cuestiones sobre el capítulo al que se dedica la docencia en seminario, previamente al desarrollo de la discusión del mismo, se pretende fomentar la adecuada preparación de la clase, así como la fijación de la idea principal y los conceptos clave a discutir. Con ello, se busca aumentar el nivel de la discusión posterior, a través de la promoción de la participación y la motivación de los y las estudiantes. Así, se aseguraría una elevación de la complejidad de las competencias del alumnado, según la taxonomía de Bloom, favoreciendo la aplicación, la síntesis y la evaluación.

PALABRAS CLAVE: gamificación, flipped classroom, clases prácticas, ludificación. 


\section{CITA RECOMENDADA:}

Crego, Jorge (2020): Kahoot como herramienta de transición en las aulas invertidas. La aplicación en la docencia en Filosofía del Derecho. En De la Torre Fernández, E. (ed.) (2020). Contextos universitarios transformadores: Boas prácticas no marco dos GID. IV Xornadas de Innovación Docente. Cufie. Universidade da Coruña. A Coruña (págs. 57-70).

DOI capítulo: https://doi.org/10.17979/spudc. 9788497497756.057

DOI libro: https://doi.org/10.17979/spudc.9788497497756

\section{ABSTRACT}

The subject of Philosophy of Law instructed at the Universidade da Coruña (UDC) includes as the lesson 7 a study of the theories of justice, based on the material of Michael J. Sandel (Harvard). That lesson is developed through seminar classes. Following the proposal of the American professor, the lesson is imparted through the methodology of inverted or flipped classroom. This article aims to assess the introduction of technological resources (Kahoot) in order to bolster the proper preparation of the class, and the identification of the main idea and key concepts to discuss. In so doing, the objective is to raise the level of the subsequent discussion, through the promotion of the engagement and motivation of the students. Thus, it will be possible to achieve an elevation of the complexity of the competences of the students, following Bloom's taxonomy, favouring application, synthesis and evaluation.

KEYWORDS: gamification, flipped classroom, practical lessons, inverted classroom. 


\section{INTRODUCCIÓN}

Michael J. Sandel, filósofo político y profesor en Harvard, lleva impartiendo su curso "Justice", una introducción a la filosofía moral y política, desde hace más de 25 años. Hasta la aparición de nuevos cursos alineados con los intereses contemporáneos', "Justice" destacó por ser el curso al que se matriculaban más estudiantes de la prestigiosa Universidadii. Según la web de Harvard, más 15.000 alumnos han cursado dicha materiaiii.

A la hora de presentar una materia que podría considerarse clásica por su contenido (por ejemplo, incluye textos escritos en el S. IV a.C. por Aristóteles), Sandel opta por un método didáctico que, bajo ciertas premisas, se ha convertido en una nueva moda: las aulas invertidas o flipped classrooms.

Pese a que en el ámbito hispanohablante la propuesta de Sandel se conoce a través de un libro de su propia autoría, con el título Justicia ¿Hacemos lo que debemos? (2000), las clases del profesor estadounidense se basan en una selección de escritos de filósofas y filósofos clásicos y contemporáneos y otros materiales seleccionados (Sandel, 2007). Las dinámicas de sus clases parten de la lectura previa de los textos propuestos y la posterior discusión dirigida por el profesoriv.

En la materia de Filosofía del Derecho (612G01026) impartida en la Unversidade da Coruña (UDC), las clases de seminario se dedican a la enseñanza de las teorías de la justicia. Para ello, se emplea el libro de Sandel antes citado, y se reproducen las dinámicas de flipped classroom ya mencionadas. El alumnado debe dedicar la semana a la lectura y preparación de uno de los capítulos del libro. Durante la sesión de seminario, dos o tres alumnos se encargan de realizar una breve exposición crítica del contenido del capítulo en cuestión y, seguidamente, se desarrolla una discusión del mismo. El objetivo de esta dinámica es doble: poder formarse en aspectos instrumentales de la asignatura, tales como la reunión e interpretación de datos relevantes para emitir juicios críticos o el manejo de la expresión y discusión propias de la materia; y, a su vez, comprender los contenidos de la teoría de la justicia en niveles superiores de las habilidades cognitivas. 
Sin embargo, durante el transcurso de los años se han observado las limitaciones de esta metodología. Este estudio se centrará en una de ellas: la posibilidad de participar en la discusión sin haber alcanzado la compresión debida de las ideas centrales del capítulo correspondiente, 0 incluso sin haber preparado los materiales. El hecho de que las diferentes teorías de la justicia se presenten a través de ejemplos prácticos objeto del debate público permite que se viertan opiniones escasamente fundamentadas, tomadas de instancias ideológicas con escaso nivel argumentativo (debates televisivos, internet, etc.). Con ello no solamente se reduce el nivel de la discusión, sino que se bloquea la posibilidad de una comprensión de las teorías de la justicia subyacentes, solo aprehensibles a través de una lectura de los materiales propuestos 0 , en todo caso, de publicaciones académicas 0 de similar entidad. La hipótesis de este trabajo es que la herramienta Kahoot puede solucionar, al menos en parte, esta limitación. De este modo, un cierto uso de la gamificación y la metodología de la flipped classroom pueden complementarse para maximizar los resultados de la docencia en seminarios.

Para defender esta hipótesis, el trabajo se estructurará del siguiente modo. Primero, se presentarán las dinámicas adoptadas para la impartición de la materia, señalando la idoneidad del método del aula invertida y su uso persistente en la docencia llevada a cabo por Michael J. Sandel. Segundo, se señalará brevemente el funcionamiento de Kahoot, reflejando las ventajas e inconvenientes que pueda tener. En tercer lugar, se señalará el modo en que se encajaría el seguimiento vía Kahoot en las dinámicas específicas del aula invertida, y cómo el primero podría fortalecer los beneficios de la segunda metodología. Seguidamente, se señalará el modo en que se podría evaluar la participación a través de Kahoot, para finalizar con unas propuestas alternativas a la inicialmente presentada.

\section{LA FLIPPED CLASSROOM COMO METODOLOGÍA DOCENTE}

Numerosos autores reconocen que la metodología de la flipped classroom o inverted classroom se ha convertido en una herramienta generalizada (Tucker, 2012; Bishop, Verleger, 
2013). También es un lugar común afirmar que el origen de esta metodología se encuentra en el trabajo de Lage, Platt y Treglia (2000) y el de Baker (2000).

Existe cierta polémica a la hora de determinar qué es la flipped classroom. La definición original de Lage es la siguiente: "Ios eventos que tradicionalmente se han tenido lugar dentro del aula ahora tienen lugar fuera del aula, y viceversa" (Lage et al., 2000: 32). Sin embargo, Bishop y Verleger la critican por ser demasiado genérica (Bishop, Verleger, 2013). Estos autores añaden dos requisitos: (i) clases de aprendizaje interactivo por grupos en el aula y (ii) aprendizaje individual basado en ordenadores fuera del aula. Pese a que la introducción del elemento informático, generalmente referido a un vídeo que viene a sustituir el modelo de las clases magistrales, es mayoritaria, considero que no resulta necesario para comprender la lógica tras la práctica de la flipped classroom ${ }^{v}$. Bajo mi punto de vista, el uso de tecnología u otros medios no altera significativamente el proceso ni el método de aprendizaje, y su inclusión parece reflejar cierta presunción optimista de que el empleo de nuevos medios tecnológicos es en sí mismo el elemento distintivo que genera los beneficios de esas nuevas metodologías.

La definición que emplearé será la siguiente: considero la flipped classroom como una metodología en la que la fase de adquisición de conocimientos se realiza fuera del aula y la fase de aplicación de conocimientos dentro de ella (Jensen et al., 2015; 0'Flaherty, Phillips, 2015), específicamente a través de aprendizaje por pares (peer-assisted learning) ${ }^{\mathrm{vi}}$.

Numerosos estudios han remarcado los beneficios de la flipped classroom (0'Flaherty, Philips, 2015), aunque existen reiteradas críticas sobre la falta de datos concluyentes (Bishop, Verleger, 2013; O’Flaherty, Philips, 2015) y la dificultad para determinar cuál es el factor determinante de los efectos positivos, dada la multiplicidad de elementos modificados por configuración de dicha práctica (Jensen et al., 2015). En el marco de las clases de Filosofía del Derecho que son objeto de este estudio, la utilidad de dicha metodología se encuentra esencialmente en la posibilidad de potenciar el aprendizaje activo en la fase de aplicación del conocimiento, con la supervisión del profesor en dicho momento. Esto permite un mayor 
seguimiento en las fases del conocimiento más elevadas en la taxonomía de Bloom (Bloom, 1984).

\section{LA INSERCIÓN DE KAHOOT PARA MAXIMIZAR LOS BENEFICIOS DE LA FLIPPED CLASSROOM}

A la hora de evaluar el impacto de las flipped classroom, varios autores apuntan a un problema grave: la nula o incorrecta preparación de los materiales previos a la clase genera niveles desiguales de preparación en el alumnado y dificulta la inserción de aquellas personas que no hayan preparado los textos en las dinámicas activas dentro del aula (0'Flaherty, Philips, 2015, 94; Sein-Echaluce et al., 2017, 610) vii. Durante la docencia de Filosofía del Derecho he observado esta amenaza, como he señalado anteriormente.

Algunos autores apuntan, en la caracterización de las dinámicas de las flipped classrooms, a la posibilidad de emplear recursos informáticos como "preguntas de pulsador" (clicker questions) como actividades realizables dentro del aula. Este tipo de mecanismos permiten una evaluación de los conocimientos de los alumnos en tiempo real (O'Flaherty, Philips, 2015, 87), de modo que son muy indicados como herramienta de transición entre las tareas fuera del aula y la propia dinámica en el aula (Fidalgo-Blanco et al., 2017, 714). Además, puede servir para mejorar la actividad fuera del aula (Sein-Echaluce et al., 2017, 611).

En la misma línea, considero que el uso de un cuestionario de evaluación en tiempo real puede cumplir una doble función: (i) implicar al conjunto del alumnado en la realización de las tareas fuera del aula, donde el profesorado no tiene la posibilidad de favorecer directamente la adquisición de conocimiento, y (ii) evaluar el grado de comprensión de las ideas clave de las lecturas recomendadas, mejorando la calidad de la dinámica dentro del aula. Precisamente por esto mismo, cumple perfectamente la función de actividad de transición entre ambos escenarios de aprendizaje. Estas funciones se ven potenciadas cuando el cuestionario de evaluación se lleva a cabo a través de sistemas de respuesta de estudiantes basado en juegos (GSRS, por sus siglas en inglés). 
Las dinámicas de "gamificación" de los espacios de enseñanza son a día de hoy una tendencia al alza. Diversos estudios han señalado su utilidad en los espacios universitarios (Sharples, 2000). Wang señala cómo el uso de GSRS impulsa la capacidad de captar la atención y motivar a los estudiantes, ya existente en cualquier recurso de obtención de respuestas del alumnado (Wang, 2014, 221). En su estudio demuestra que los GSRS, en concreto Kahoot, mejoran las dinámicas en el aula (mayor concentración) e impulsan la atención, la motivación y el aprendizaje percibido por los alumnos. Además, este estudio descarta la posibilidad de que un uso continuado del GSRS disminuya notablemente su utilidad, e incluso señala que una periodicidad de un uso semanal es adecuada. Una evaluación exhaustiva de la literatura existente hasta 2007 puede encontrarse en Kay y LeSage (2009).

Entre los diferentes GSRS se ha optado por emplear Kahoot por ser el que la literatura destaca como más eficaz (Göskun, Gürsoy, 2019), así como por las experiencias positivas de otros docentes de la Facultad de Derecho de la UDC (Rodríguez-López, 2019). Dada la popularidad de la aplicación, basta una breve explicación de su funcionamiento. Kahoot permite esencialmente la elaboración de preguntas con cuatro posibles respuestas, de las cuales una ha de ser la correctaviii. Para poder emplearlo en el aula, el o la profesora debe emplear una pantalla visible por todo el alumnado, donde se reflejarán las preguntas y las respuestas. Cada alumno 0 alumna deberá emplear un dispositivo móvil en el que deberá seleccionar la respuesta que considera correcta. Existe una aplicación de móvil de Kahoot, pero también puede accederse al juego a través de internet. Para cada pregunta se fija un tiempo de respuesta. Cuando transcurre el tiempo previsto, se muestra la respuesta correcta, y el número de personas que seleccionó cada una de las respuestas. Esto permite evaluar el grado de comprensión del contenido de la pregunta. Finalmente, basándose en el tiempo en que se ha tardado en responder, se muestra una clasificación de las cinco personas que han obtenido más puntos. Tras finalizar todas las preguntas, cada alumno 0 alumna recibe sus resultados personales, y el o la profesora recibe una tabla con toda la información. 
La propuesta docente del uso de Kahoot es por tanto la siguiente: se presentarán tres preguntas a través de dicha aplicación al comienzo de las dinámicas dentro del aula. Entre ellas se incluirá una pregunta dirigida a comprobar si el alumnado ha leído el material a preparar fuera del aula y dos a evaluar hasta qué punto se han comprendido las ideas esenciales que se presentan en el mismo. Una vez obtenidos los resultados, se dará inicio a la presentación crítica por parte del grupo encargado, y finalmente se desarrollará la discusión.

El efecto esperado de la introducción de Kahoot, habida cuenta de la metodología de flipped classroom que se desarrolla en las clases prácticas de Filosofía del Derecho es el siguiente. A través de la competitividad que genera dicha aplicación, se pretende que el alumnado encuentre motivos añadidos para la preparación de los materiales fuera del aula (Wang, 2014, 224). La perspectiva de poder obtener algunos puntos extra, así como la posibilidad de verse reflejado en posiciones altas de una clasificación de los conocimientos del alumnado, pueden servir para reducir el principal problema que se ha observado en el desarrollo de las clases con la dinámica de flipped classroom descrita. Además, al poder comprobar los conocimientos adquiridos fuera del aula, tanto el o la profesora como el grupo encargado de la presentación podrán identificar sobre qué elementos incidir en su explicación. De este modo, se podrá afrontar el debate posterior en mejores condiciones. Los estudios al respecto señalan que los efectos en la motivación, la comprensión de los materiales y la participación aumentan de manera notable (Wang, 2014, 224). Estos tres elementos son precisamente los que se han identificado como esenciales pare mejorar las dinámicas de las clases objeto de estudio.

Como se ha apuntado, el empleo de la aplicación Kahoot servirá además para impulsar el desarrollo de las habilidades más elevadas de la taxonomía de Bloom. Las habilidades más básicas (recordar y comprender) se reservan para las tareas fuera del aula. Gracias al uso de Kahoot se solventarán dudas y se asegurará una correcta realización de dichas fases del aprendizaje. Con ello, habilidades más elevadas, como la capacidad de aplicar conocimiento a nuevas situaciones, la evaluación de las ideas o la creación de conocimiento podrán realizarse en el aula, con apoyo del profesor o profesora y a partir de unos conocimientos más refinados. 


\section{PROPUESTA DEL MODO DE CALIFICACIÓN DEL JUEGO CON KAHOOT}

Para que la propuesta funcione, resulta fundamental configurar un método de puntuación efectivo, que asegure el interés del alumnado y también apoye los efectos del empleo del juego en lo referido a la clarificación del contenido.

Para ello, es necesario tener en cuenta algunas limitaciones derivadas de la estructuración de las clases. Generalmente, en los estudios de grado, las clases prácticas se dividen en tres grupos reducidos. En el caso del doble grado de Derecho y ADE, la división es en dos grupos. Para evitar que los grupos que tienen la clase con posterioridad puedan conocer las preguntas, es necesario elaborar un trío de preguntas para cada grupo. Esto genera un problema de comparación entre grupos. Sin embargo, es el único modo de asegurar que los efectos de la metodología no se diluyen.

Como se ha dicho, se elaborarán tres preguntas para cada grupo. Dos de ellas se centrarán en la aclaración de ideas centrales de los capítulos, mientras que una tercera tendrá como objetivo asegurar que se ha leído el capítulo correspondiente. Para ello, se seleccionará algún ejemplo 0 anécdota cuya respuesta correcta sea improbable sin haber preparado el capítulo.

El método de Kahoot, que puntúa tanto el acierto como la velocidad de respuesta resulta muy oportuno para evitar otros problemas que podrían darse. Debido a este modo de puntación resulta improbable que se pueda producir una colaboración sistemática entre el alumnado. En cualquier caso, cada alumno 0 alumna estará tentado a responder cuanto antes, de modo que incluso si quisiese trasladar su respuesta a un o una compañera, lo hará después de haber obtenido una mayor puntuación por responder con mayor celeridad. De este modo se asegura que aquellas personas que han preparado mejor la clase sean las que obtengan mejores puntuaciones, y que las que no lo han hecho sean relegadas a las últimas posiciones de la clasificación, manteniéndose la presión competitiva dirigida a preparar mejor la materia la semana siguiente.

Debido a que el libro cuenta con diez capítulos, el total de preguntas respondidas por cada alumno 0 alumna será de 30 . Para recompensar el esfuerzo del alumnado y mantener un 
incentivo durante las diez sesiones, se premiará con un máximo de un punto sobre el total de 10 dependiendo de la posición ocupada en cada grupo reducido. Las diez primeras personas de cada grupo obtendrán puntos a sumar a la nota final. La puntuación se repartirá del siguiente modo: 1 punto a la primera persona clasificada, 0,9 a la segunda, 0,8 a la tercera, y así sucesivamente hasta la décima, que recibirá un 0,1. Como criterio extra, se establecerá que la puntuación solamente se sumará en caso de haber respondido correctamente al menos al $75 \%$ de las preguntas. En caso de que una de las 10 primeras clasificadas no obtenga ese porcentaje, los puntos no se repartirán a las clasificadas inferiores. Al puntuar a cada grupo por separado se evitarán conflictos de comparación. Al finalizar todas las sesiones se mostrará una tabla conjunta con los resultados de toda la clase.

\section{POSIBLES ALTERNATIVAS AL MODELO PROPUESTO}

La evaluación de algunas propuestas existentes en la literatura sobre las flipped classrooms y el uso de GSRS se han extraído una serie de dinámicas cuya utilización puede ser interesante. Pese a que no se incluyen en esta primera propuesta, resulta oportuno tenerlas en cuenta. A la luz de futuras experiencias se podrá evaluar su inserción.

En su artículo seminal sobre las flipped classrooms, Lage, Platt y Treglia describen su propuesta de trabajo fuera del aula como lecturas sobre un tema que, en su caso, se complementan con un vídeo sobre la temática. Se anima al alumnado a verlo, pero no parece ser el elemento central de la propuesta (Lage et al., 2000, 32-33). En el futuro se podría explorar la posibilidad de grabar pequeños vídeos explicativos, que se pondrían a disposición del alumnado. Debido a que las dinámicas dentro del aula se inician con una presentación por parte de grupos de alumnos y alumnas, para que estos vídeos no interfieran en la preparación de dicha presentación, 0 terminen por substituirla, es fundamental que se centren en elementos muy específicos del capítulo, o cuestiones preliminares que sin explicar las ideas centrales del mismo puedan servir para comprenderlas. 
Por su parte, Wang presenta una alternativa interesante. Señala que el mejor modo de optimizar el aprendizaje con GSRS es exigiendo al alumnado que se encargue de la elaboración de las preguntas. De este modo, podría solicitarse al grupo encargado de la exposición del capítulo la elaboración de las preguntas, que deberían seguir las características exigidas por el o la profesora. Esto ofrecería un modo de exigir al alumnado que demuestra otro tipo de habilidades cognitivas, a través de la capacidad para identificar las ideas centrales del capítulo o los puntos críticos del mismo.

Por otro lado, esta alternativa también permitiría un mayor seguimiento por parte del profesorado. Se podría plantear que las preguntas deberán trasladarse con anterioridad, para su evaluación y aprobación previa al ejercicio. De este modo, se añadiría un momento de evaluación que serviría para corregir incomprensiones y para valorar más pormenorizadamente a las dos 0 tres personas encargadas del capítulo. Esto podría ser muy positivo, pues ayudaría a identificar de manera individual quién ha llevado el peso de la preparación de los materiales, así como a elevar la comprensión del alumnado a través de precisiones o discusiones durante la selección definitiva de las preguntas.

\section{REFERENCIAS}

Baker, J.W. (2000). The 'Classroom Flip’: Using Web Course Management Tools to Become the Guide by the Side. En J.A. Chambers (ed.), Selected Papers from the $11^{\text {th }}$ International Conference on College Teaching and Learning (pp. 9-17). Jacksonville: Community College at Jacksonville.

Bishop, J.L., Verleger, M.A. (2013). The Flipped Classroom: A Survey of the Research. ASEE National Conference Proceedings, 30(9), pp. 1-18.

Bloom BS (1984). Taxonomy of Educational Objectives. Boston: Allyn and Bacon.

Göskun, D.0., Gürsoy, G. (2019). Comparing success and engagement in gamified learning experiences via Kahoot and Quizizz. Computers \& Education, 135, pp. 15-29. 
Jensen, J.L., Kummer, T.A., Godoy, P.D.d.M. (2015). Improvements from a Flipped Classroom May Simply Be the Fruits of Active Learning. CBE - Life Sciences Education, 14(1), pp. $1-12$.

Kay, R.H., LeSage, A. (2009). Examining the benefits and challenges of using audience response systems: A review of the literature. Computers \& Education, 53, pp. 819-827. Lage, M.J., Platt, G.J., Tregilia, M. (2000). Inverting the Classroom: A Gateway to Creating an Inclusive Learning Environment. Journal of Economic Education, 31(1), pp. 30-43.

0'Flaherty, J., Phillips, C. (2015). The use of flipped classroom in higher education: A scoping review. Internet and Higher Education, 25, pp. 85-95.

Papadopoulos, C., Santiago-Román, A. (2010). Implementing an inverted classroom model in engineering statics: Initial results. En Proceedings of the ASEE Annual Conference \& Exposition. Louisville, Kentucky, pp. 15.679 .1 - 15.679.27.

Rodríguez-López, S. (2019). Valoracións por parte do alumnado da utilización de ferramentas virtuais de resposta de audiencia no Grao en Dereito. En Congreso de innovación docente no ámbito xurídico. Coruña.

Sandel, M.J. (2011). Justicia. ¿Hacemos lo que debemos? Barcelona: Debate.

---- (2007). Justice: A Reader. Oxford: Oxford University Press.

Sein-Echaluce, M.L., Fidalgo-Blanco, Á., García-Peñalvo, F.J. (2017). Trabajo en equipo y Flip Teaching para mejorar el aprendizaje activo del alumnado. En Sein-Echaluce, M.L., Fidalgo-Blanco, Á., García-Peñalvo, F.J. IV Congreso Internacional sobre Aprendizaje, Innovación y Competitividad-CINAIC 2017 (4-6 Octubre 2017, Zaragoza, España). Zaragoza: Servicio de Publicaciones Universidad de Zaragoza, pp. 610-615.

Sharples, M. (2000). The design of personal mobile technologies for lifelong learning. Computers \& Education, 34(3-4), pp. 177-193.

Tucker, B. (2012). The Flipped Classroom. Online instruction at home frees class time for learning. Education Next, 12(1), pp. 82-83. 


\footnotetext{
i https://www.classcentral.com/report/harvard-cs50-new-courses/

ii https://www.thecrimson.com/article/2007/9/26/sandel-wins-enrollment-battle-justice-triumphs/

iii https://online-learning.harvard.edu/instructor/michael-j-sandel

iv Las clases que Sandel impartió en el curso 2005 están disponibles en Youtube. Fueron en su día las primeras clases de Harvard publicadas a través de este medio. Además, la plataforma de MOOCs edX ofrece este mismo curso. https://www.youtube.com/watch? $v=k B d f c R-8 h E Y \& l i s t=P L 30 C 13 C 91 C F F E F E A 6$

$\checkmark$ Algunos autores, como reconocen Bishop y Vergler, no reconocen este requisito (Papadopoulos, Santiago-Román, 2010; O’Flaherty, Phillips, 2015).

vi Esta definición otorga el mayor peso a la alteración de las fases de conocimiento, elimina la referencia al uso de tecnologías e introduce la exigencia de interacción entre el alumnado en el seno del aula.

vii Bishop y Verleger señalan también este problema. En su caso, además, Io introducen como argumento para descartar que se pueda considerar como flipped classroom metodologías que asignan lecturas en lugar de vídeos como tareas fuera del aula (Bishop, Verleger, 2013, nota 2). Este argumento, sin embargo, como se ha apuntado, es demasiado pobre como para determinar la definición de dicho método. Primero, porque lo mismo puede suceder con un vídeo. Segundo, porque dicho problema debe ser objeto de evaluación y solución, no motivo para categorizar la práctica en sí.

viii También permite, en su versión gratuita, preguntas de verdadero o falso. Sin embargo, estas no se consideran tan útiles.
} 
